\title{
Effects of Sphingosine, Staurosporine, and Phorbol Ester on Neurites of Rat Sympathetic Neurons Growing in Compartmented Cultures
}

\author{
Robert B. Campenot, Anil H. Walji, and Dwight D. Draker \\ Department of Anatomy and Cell Biology, Faculty of Medicine, University of Alberta, Edmonton, Alberta, \\ Canada T6G 2H7
}

Local application of sphingosine (1-10 $\mu \mathrm{M})$, an inhibitor of protein kinase C, to NGF-supplied, distal neurites of rat sympathetic neurons in compartmented cultures caused their retraction and/or degeneration within $24 \mathrm{hr}$. This effect was specific for distal neurites because sphingosine (even at $100 \mu \mathrm{M})$ applied to cell bodies and/or proximal neurites did not destroy these regions of the cells, and their distal neurites continued to elongate. However, effects of other agents suggest that the retraction/degeneration observed in distal neurites directly exposed to sphingosine is not mediated by inhibition of protein kinase C: application of staurosporine, another inhibitor of protein kinase $\mathrm{C}$, to distal neurites did not cause retraction or degeneration; treatment of neurons for 24 or more hours with $2 \mu \mathrm{M}$ phorbol 12-myristate 13acetate (PMA), used to downregulate protein kinase $\mathrm{C}$ activity, slowed neurite extension about $50 \%$, but did not cause degeneration; and neurons pretreated with PMA still displayed retraction/degeneration of neurites when they were subsequently exposed to sphingosine. Also, replacement of NGF supplied to distal neurites with anti-NGF IgG did not cause retraction/degeneration of neurites within $1 \mathrm{~d}$, suggesting that the effect of sphingosine did not arise by interference with the action of NGF.

The specificity of the sphingosine-induced retraction/degeneration for distal neurites suggests that this effect operates via specific mechanisms in distal neurites that can trigger their retraction/degeneration. Such mechanisms could play important roles in nerve growth inhibition, nerve fiber retraction, and degeneration that occur normally in the nervous system and in response to injury and disease. Also, the ability of neurites to grow in the presence of PMA suggests that neurite growth is not dependent upon the activity of protein kinase $C$. However, the reduced rate of neurite extension in the presence of PMA suggests that chronic PMA treatment may affect mechanism(s) that can modulate neurite growth.

\footnotetext{
Received Sept. 17, 1990; revised Nov. 21, 1990; accepted Nov. 27, 1990.

This work was supported by an establishment grant from the Alberta Heritage Foundation for Medical Research and by a program grant from the Medical Research Council of Canada. Richard Murphy generously supplied the $2.5 \mathrm{~S} \mathrm{NGF}$ and the affinity-purified sheep anti-NGF IgG. We thank Grace Dotto for technical assistance and Gregory Morrison for photographic work. We also thank Ann Acheson, David Brindley, and Richard Murphy for their helpful comments on the manuscript.

Correspondence should be addressed to Robert B. Campenot, Department of Anatomy and Ccll Biology, Faculty of Mcdicinc, University of Albcrta, Edmonton, Alberta, Canada T6G 2H7.

Copyright (C) 1991 Society for Neuroscience $0270-6474 / 91 / 111126-14 \$ 03.00 / 0$
}

NGF stimulates neurite production in sympathetic neurons and several other types of neurons, but the mechanisms that link NGF receptor activation to neurite growth remain elusive. $\mathrm{Re}$ cent work has called into question earlier suggestions that cAMP plays a role (Richter-Landsberg and Jastorff, 1986; Rydel and Greene, 1988). Another possibility is involvement of protein kinase $\mathrm{C}(\mathrm{PKC})$. NGF treatment activates PKC in PC12 cells (Heasley and Johnson, 1989), and the sprouting of neurites by PC12 cells is suppressed by sphingosine, a membrane sphingolipid known to inhibit PKC activity (Hall et al., 1988). However, NGF has effects other than just promoting neurite growth (see Yankner and Shooter, 1982), and sphingosine has effects other than just inhibiting PKC (Faucher et al., 1988; Winicov and Gershengorn, 1988; Hannun and Bell, 1989; Reinhold and Neet, 1989). In fact, results of experiments in which PKC activity is blocked by means other than sphingosine lead to a different conclusion. Phorbol esters are well-known short-term activators of PKC, but long-term treatment results in its downregulation (Ballester and Rosen, 1985). Treatment of PC12 cells for $24 \mathrm{hr}$ with $1 \mu \mathrm{M}$ phorbol 12-myristate 13-acetate (PMA) resulted in the downregulation of $\mathrm{PKC}$, virtually eliminating its activity (Matthies et al., 1987; Heasley and Johnson, 1989). However, PC12 cells pretreated with PMA nonetheless sprouted neurites in response to NGF, and did so with the same frequency as untreated cells (Reinhold and Neet, 1989). This suggests that PKC activity is not necessary for NGF to elicit neurite sprouting by $\mathrm{PC} 12$ cells. Consequently, the block of neurite sprouting by sphingosine does not appear to arise through block of PKC activity. Some other action of sphingosine would appear to be responsible.

The experiments reported in the present study were undertaken in light of this previous work but from the point of view that neurite growth might be controlled differently in neurons than in PC1 2 cells; the latter are a line of adrenal tumor cells that cease dividing and adopt a neuronal phenotype when given NGF (Greene and Tischler, 1976). Thus, it seemed potentially informative to undertake studies with sympathetic neurons cultured from newborn rats. As with PC12 cells, PKC activity in cultured rat sympathetic neurons is downregulated in response to long-term exposure to $1 \mu \mathrm{M}$ PMA; strong suppression of PKC activity occurred within $24 \mathrm{hr}$ after PMA administration (Matthies et al., 1987). Therefore, the PMA paradigm for eliminating PKC activity should be an effective experimental tool for investigating the role of $\mathrm{PKC}$ in the growth of nerve fibers.

In the present study the effects of PMA, sphingosine, and another inhibitor of PKC, staurosporine, were investigated. Neurite growth was measured in compartmented cultures in 
which cell bodies reside in a proximal compartment and neurites grow along a series of collagen tracks, penetrate silicone grease barriers, and elongate within distal compartments (Fig. 1). In this system the cell bodies and proximal neurites can be exposed to culture media of different composition than distal neurites (Campenot and Draker, 1989), making it possible to experimentally distinguish local influences within distal neurites that control growth from those involving the cell body. Also, neurites can be mechanically removed from distal compartments, they begin regenerating within $1 \mathrm{~d}$ (Campenot, 1982a), and the progress of regenerating neurites along the tracks can be conveniently measured over several days. The sustained neurite elongation that is evaluated by this method provides a different measure of growth from the percent of cell bodies that sprout neurites that has been used with PC12 cells. Because neurite growth continues long after sprouting from the cell body has presumably ceased, it is likely that sustained neurite growth is regulated differently from initial sprouting. For all these reasons, an investigation of the pharmacology of neurite growth using sympathetic neurons in compartmented cultures was considered likely to yield new information.

\section{Materials and Methods}

General culturing procedures. Principal neurons from superior cervical ganglia of newborn rats (Sprague-Dawley, supplied by the University of Alberta Farm) were dissociated and plated into 3-compartmented culture dishes at a density of about 1 ganglion per dish. The general culturing procedures and formulations were similar to those used previously (Campenot, 1982a), essentially following Hawrot and Patterson (1979). One departure from previous practice was the use of a combined enzymatic and mechanical dissociation procedure for the neurons. Twenty superior cervical ganglia were dissected from 10 newborn rats as previously described, placed with forceps into a $15-\mathrm{ml}$ disposable centrifuge tube, and washed to the bottom with phosphate-buffered saline (PBS). All but $1.8 \mathrm{ml}$ of PBS was pipetted off, and $200 \mu \mathrm{l}$ trypsin (Calbiochem, La Jolla, CA) stock (1\% in PBS) was added. The tube was incubated for $20 \mathrm{~min}$ in a $37^{\circ} \mathrm{C}$ water bath. Then, $100 \mu \mathrm{l}$ DNAase (Sigma, St. Louis, MO) stock $(0.2 \mathrm{mg} / \mathrm{ml}$ PBS) was added, and the ganglia were mixed gently with a sterile, cotton-plugged Pasteur pipette to separate the clumps into single ganglia. The ganglia were then rinsed twice with PBS and once with L-15 air medium containing $10 \%$ rat serum. After removal of this last rinse, $7 \mathrm{ml}$ of plating medium was added, and the medium along with the ganglia was pipetted with a Pasteur pipette into a $60-\mathrm{mm}$ culture dish. With the aid of a dissecting microscope, the ganglia were then placed into a lab-made cell dissociator. The dissociator consisted of a 1-ml glass syringe with the barrel cut off just before the neck that would ordinarily receive the hypodermic needle. A fine stainless-steel screen had been cemented over the end of the barrel with epoxy cement so that the plunger could be used to gently grind the ganglia against the screen. An opening had been cut in the side of the syringe barrel near the screen to allow loading of ganglia when the plunger was slightly withdrawn. Gentle turning of the plunger against the ganglia for $2-5 \mathrm{~min}$ resulted in the release of cell suspension. The suspension was then pipetted into a centrifuge tube and spun in an International Equipment $\mathrm{Co}$. clinical centrifuge for $3 \mathrm{~min}$ at setting 3 $(1160 \mathrm{rpm})$. The supernatant was pipetted off, and the cells were resuspended into $2 \mathrm{ml}$ of culture medium and plated into compartmented cultures as previously described (see below and Campenot, 1979, 1987). The combined enzymatic/mechanical dissociation procedure yields about twice as many neurons as the mechanical procedure alone so that a plating density of about 1 ganglion per dish was used instead of 2 . Cultures were maintained in a tissue culture incubator at $37^{\circ} \mathrm{C}$ and $5 \%$ $\mathrm{CO}_{2}$.

Compartmented cultures. Compartmented cultures were constructed essentially as previously described (Campenot, 1979). Briefly, collagen extracted from rat tail tendons was dried onto $35-\mathrm{mm}$ Falcon tissue culture dishes to provide a culture substratum (see Hawrot and Patterson, 1979). A series of collagen tracks was then formed between parallel scratches made in the dish floor from which the dried collagen had been scraped away. A Teflon divider (Protomatic Inc., Dexter, MI) was then seated to the surface with silicone grease. Neurons were plated by using a $1-\mathrm{ml}$ disposable syringe with a 22 -ga needle to deposit the neurons suspended in methylcellulose-thickened culture medium into the dishes. In center-plated cultures (Fig. $1 b$ ), about $100 \mu \mathrm{l}$ of suspension was deposited into the slotlike center compartment of each dish, and the neurons were allowed to settle onto the substratum overnight. The next day, the outer perimeter of the dish was given about $1 \mathrm{ml}$ of medium. Side compartments each contained about $0.5 \mathrm{ml}$ of medium. The neurons settled on the substratum of collagen tracks, and neurites elongated on these tracks, crossing under the silicone grease barriers to enter left and right compartments. In some experiments, left-plated cultures were used (Fig. $1 \mathrm{c}$ ). This required putting down a perimeter of silicone grease in the left compartment to confine the cell bodies to regions of the tracks near the barrier. Center and right compartments were filled with medium, and a drop of cell suspension (about $100 \mu \mathrm{l}$ ) was placed in the left compartment within the grease perimeter and resting against the Teflon divider. The next day, after the cells had settled on the substratum, the left compartment was filled with medium.

In some experiments, cultures were neuritotomized and regeneration observed. Neuritotomy was performed by washing away the neurites in left and right compartments with a jet of sterile, distilled water delivered with a syringe through a 22-ga needle. The water was aspirated and the wash repeated twice more, then fresh culture medium was added. This procedure reliably removes virtually all visible traces of neurites from the side compartments. In one experiment, a similar procedure was used to remove the cell bodies and proximal neurites from the center compartments.

Neurite extension on individual collagen tracks was measured using a digital micrometer (Ono Sokki), with a resolution of $2 \mu \mathrm{m}$, mounted so as to measure stage movements of the Nikon Diaphot inverted microscope. Time-lapse video observations were made using a Zeiss ICM 405 inverted microscope with a heating stage set at $37^{\circ} \mathrm{C}$. During video sessions, cultures were maintained with L-15 air medium. An MTI NC$67 \mathrm{M}$ video camera, an MTI HR-2000 monitor, and a JVC BR-9000U time-lapse video recorder were used. All observations were made using phase-contrast optics.

Culture media and experimental treatments. L-15 medium without antibiotics (Gibco Laboratories, Grand Island, NY) was supplemented with the prescribed additives, including bicarbonate and methylcellulose. Rat serum $(2.5 \%)$ and ascorbic acid $(1 \mathrm{mg} / \mathrm{ml})$ were supplied only in medium given to compartments that contained cell bodies. Culture medium was routinely changed every 3-6 d. Non-neuronal cells were eliminated by an initial exposure to medium containing $10 \mu \mathrm{M}$ cytosine arabinoside. Initially, all 3 compartments were supplied with $8 \mathrm{nM} 2.5 \mathrm{~S}$ NGF to allow cell survival and to allow neurite growth to occur in all compartments. In center-plated cultures, NGF was discontinued in the center compartments between days $6-10$. Neurons survive removal of NGF from the center compartments, and subsequent neurite growth is largely confined to the NGF-containing side compartments (Campenot, 1982a, 1987). Except where indicated, NGF was discontinued in the left and center compartments of left-plated cultures between days 6-10 in order to confine neurite growth to the right compartments. In experiments to determine the effect of NGF removal, medium without NGF was used to which $24 \mathrm{~nm}$ affinity-purified sheep anti-NGF IgG ( $3 \times$ the concentration of NGF in medium) had been added to neutralize any residual NGF that may not have been removed when the medium was changed. In all experiments, the culture medium used for experimental and control groups was identical, that is, from the same batch, except for the experimental variable.

Experimental treatments were accomplished as follows: Sphingosine (Sigma) was dissolved in $95 \%$ ethanol to make a 20 -mM stock, and this was added to medium for the desired final concentration. For most experiments 8 or $10 \mu \mathrm{M}$ sphingosine was used, and the ethanol concentration was $0.05 \%$ or less. The highest concentration of sphingosine was $100 \mu \mathrm{M}$, in which case ethanol was $0.5 \%$. In all experiments, controls had ethanol added at concentrations matching those in sphingosinecontaining medium. The decreasing concentrations of sphingosine for the dose-response experiment were achieved by serial dilution, as were the decreasing concentrations of ethanol in control media. Ceramide and palmitate were from Sigma, and stock solutions were prepared in $95 \%$ ethanol at $3 \mathrm{mg} / \mathrm{ml}$ and $20 \mathrm{~mm}$, respectively.

Phorbol 12-myristate 13-acetate (PMA; Sigma) was dissolved in dimethylsulfoxide (DMSO) to make a 2-mM stock solution, and this was added to culture medium to make a final concentration of $2 \mu \mathrm{M}$. Thus, the concentration of DMSO was $0.1 \%$, and control compartments were given a matched aliquot of DMSO without PMA. 


\section{a Compartmented culture}

Figure 1. Schematic of a compartmented culture. $a$ illustrates an entire culture, and $b$ is an enlargement of a single track in a culture with neurons plated in the center compartment. Neurites extend to the left and right, under silicone grease barriers, and into the separate fluid environments of left and right compartments. $c$ is an enlargement of a single track with neurons plated in the left compartment. Neurites cross under a silicone grease barrier, span the center compartment, cross under a second barrier, and extend within the right compartment. The tracks are formed on the collagen-coated floor of a $35-\mathrm{mm}$ plastic tissue culture dish between a series of parallel scratches from which the dried collagen substratum has been scraped away. Each track is about $200 \mu \mathrm{m}$ wide, the center compartments are about $1 \mathrm{~mm}$ wide, and the barriers are about $0.5 \mathrm{~mm}$ wide. One culture can contain up to 20 tracks occupied by neurons. Neurite extension along the tracks is measured with a stage micrometer out to about $5 \mathrm{~mm}$ into left and right compartments, where the neurites reach the ends of the tracks and become off scale for measurement.

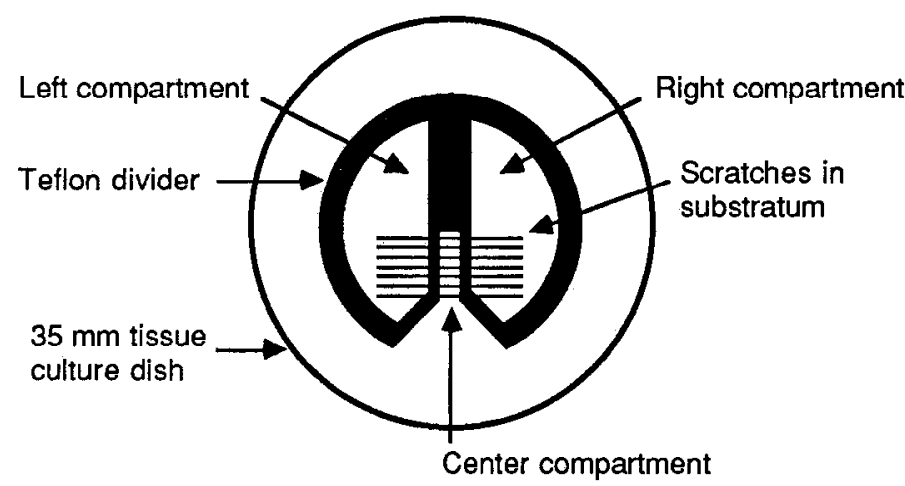

b Enlargement of a single track with neurons plated in the center compartment

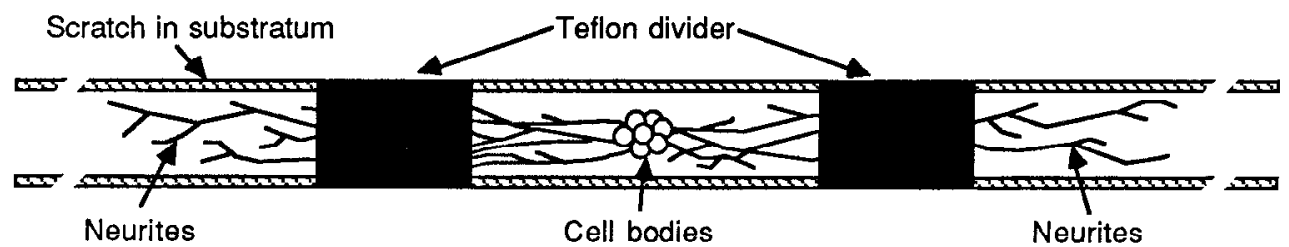

C Enlargement of a single track with neurons plated in the left compartment

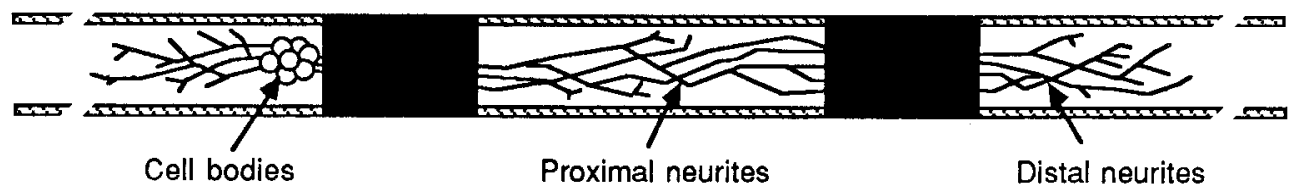

Staurosporine was from Kamiya Biomedical Company (Thousand Oaks, CA) and 3 different stock solutions were used in different experiments. For the first experiment, in which the desired final concentration was $3 \mathrm{nM}$, a 2-mM stock of staurosporine in DMSO was prepared and first diluted to $2 \mu_{\mathrm{M}}$ staurosporine by adding distilled water. Then, this was aliquoted into medium for a final concentration of $3 \mathrm{nM}$ staurosporine. The final medium contained $1.5 \times 10^{-40}$ DMSO, and control medium was made up with this concentration of DMSO alone. In the second experiment, in which the final concentration of staurosporine was $100 \mathrm{nM}$, a $20-\mu \mathrm{M}$ staurosporine stock in DMSO was used, yielding a final DMSO concentration of $0.5 \%$, and control medium was made accordingly. In the last 2 experiments, in which staurosporine was used at a final concentration of $1 \mu \mathrm{M}$, a 2-mM stock in DMSO was added, giving a final DMSO concentration of $0.05 \%$. Lower concentrations of staurosporine were achieved by serial dilution.

\section{Results}

\section{General plan of the experiments}

Compartmented cultures of 2 configurations were used: centerplated cultures in which the cell bodies of sympathetic neurons from superior cervical ganglia of newborn rats were located in the center compartments (Fig. $1 b$ ) and left-plated cultures in which the cell bodies were located in the left compartments (Fig. $1 c)$. Cultures were initially provided with medium containing $8 \mathrm{nM} 2.5 \mathrm{~S}$ NGF in all compartments. The supply of NGF to center compartments of center-plated cultures was discontinued after 6-10 d to concentrate subsequent neurite growth into the NGF-containing side compartments (Campenot, 1982a, 1987). Except where specifically indicated, center-plated cultures were maintained with NGF supplied in left and right compartments, but not in the center compartments, throughout the experiment. For the same reason, the supply of NGF to left and center compartments of left-plated cultures was discontinued after 6$10 \mathrm{~d}$, except where specifically indicated. Cultures were maintained for 8-33 d before the start of experimentation.

To begin an experiment, the distal ncurites in the left and right compartments of center-plated cultures (or the distal neurites in the right compartments of left-plated cultures) were mechanically removed with a jet of sterile, distilled water delivered through a syringe (neuritotomy; see Materials and Methods). Neurites were allowed to regenerate for several days before the experimental treatment was begun. In this way the effects of treatment on neurite extension and survival could be observed.

\section{Sphingosine causes regression of neurites}

To determine the response of growing neurites to sphingosine, neurites in the left and right compartments of center-plated cultures were removed on day 10.0 and allowed to regenerate 
for $3.0 \mathrm{~d}$, following which neurites in the right compartments were exposed to 3 different concentrations of sphingosine. Two cultures each were given $1 \mu \mathrm{M}, 10 \mu \mathrm{M}$, and $100 \mu \mathrm{M}$ sphingosine. As controls, left compartments in each of these cultures were given an amount of ethanol vehicle equal to that given in the right $(0.005 \%, 0.05 \%$, and $0.5 \%$, respectively). Measurements taken just before and $1.0 \mathrm{~d}$ after sphingosine administration showed that neurites in the right compartments, which had extended $4.85 \mathrm{~mm}(\mathrm{SEM}= \pm 0.13 \mathrm{~mm} ; N=36$ ), were virtually eliminated by exposure to $100 \mu \mathrm{M}$ sphingosine. Neurites remained on only 1 track and extended just $80 \mu \mathrm{m}$. More residual neurites remained in compartments given $10 \mu \mathrm{M}$ sphingosine, with neurite extension decreasing from a mean of $5.04 \pm 0.12$ $\mathrm{mm}(N=36)$ before treatment to $0.6 \pm 0.10 \mathrm{~mm}$ the next day. A much smaller but definite effect was displayed by neurites exposed to $1 \mu \mathrm{M}$ sphingosine. These lost relatively small regions of their distal ends, decreasing from $3.90 \pm 0.13 \mathrm{~mm}$ to 3.47 $\pm 0.24 \mathrm{~mm}(N=34)$. Control neurites in left compartments of all cultures were unaffected and continued to elongate during the treatment period.

To confirm this response of neurites to sphingosine, 2 centerplated cultures were neuritotomized on day 32.9 and supplied with NGF in all compartments. Measurements $3.0 \mathrm{~d}$ after neuritotomy showed ncurites extending about equal distances in the left and right compartments (Fig. 2a). Right compartments of both cultures were then given medium containing $8 \mu \mathrm{M}$ sphingosine, and the left, control compartments were given medium supplied with ethanol vehicle $(0.04 \%)$. Within $1 \mathrm{~d}$, neurites given sphingosine had regressed, losing about $0.70 \mathrm{~mm}$, while control neurites continued to elongate. It is also apparent in Figure $2 a$ that the neurites in compartments given sphingosine began to extend again within 2 or $3 \mathrm{~d}$ after sphingosine administration. This occurred even though the sphingosine-containing medium was still present. Subsequent experiments (see below) indicated that sphingosine-containing medium loses its ability to cause neurite retraction/degeneration with time in culture. Thus, the recovery of neurite growth observed after sphingosine administration probably does not represent accommodation of the neurons to sphingosine. However, these results do show that ncurite growth recovers from the effects of sphingosine.

In order to determine whether or not the sphingosine-induced regression was an entirely nonspecific response, the effects of ceramide and palmitic acid were investigated. Sphingosine is the lysosphingolipid derivative of ceramide formed by removal of the amide-linked fatty acyl chain. Thus, ceramide carries 2 hydrophobic tails (sphingosine has 1) and lacks the positive charge on the amino group. Palmitic acid was used to detect nonspecific effects of an acyl chain. Four center-plated cultures were neuritotomized on day 15.0 and allowed to regenerate 3.0 d with unaltered conditions. Then, 2 cultures were given $8 \mu \mathrm{M}$ sphingosine in the right compartments and $2.4 \mu \mathrm{g} / \mathrm{ml}$ ceramide in the left compartments (because chain length varies in ceramide, a wt $/ \mathrm{ml}$ equivalent to sphingosine was used). The remaining 2 cultures were given $8 \mu \mathrm{M}$ sphingosine in the right compartments and $8 \mu \mathrm{M}$ L-palmitic acid in the left compartments. Most of the length of the neurites in the right compartments was lost within $1 \mathrm{~d}$, while neurites in control compart-

Figure 2. Neurite extension in cultures given $8 \mu \mathrm{M}$ sphingosine after 3 d regeneration. Error bars are the SEM and, in some cases, are included within the symbols. $a$, Neurite extension in right and left compartments a

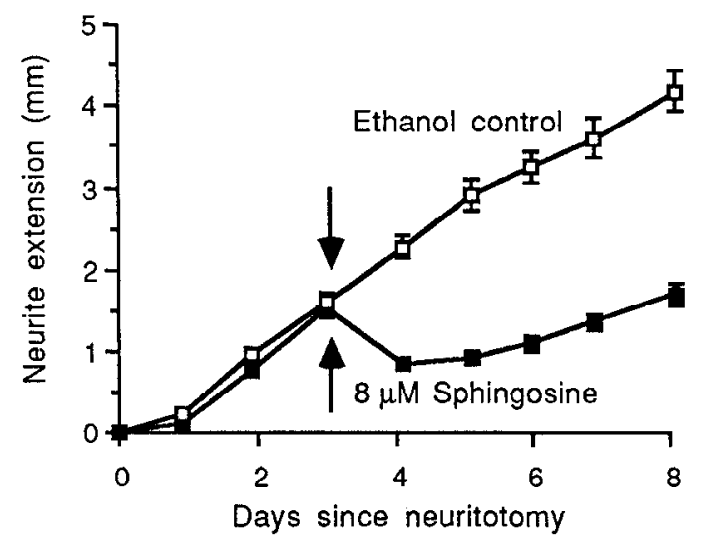

b

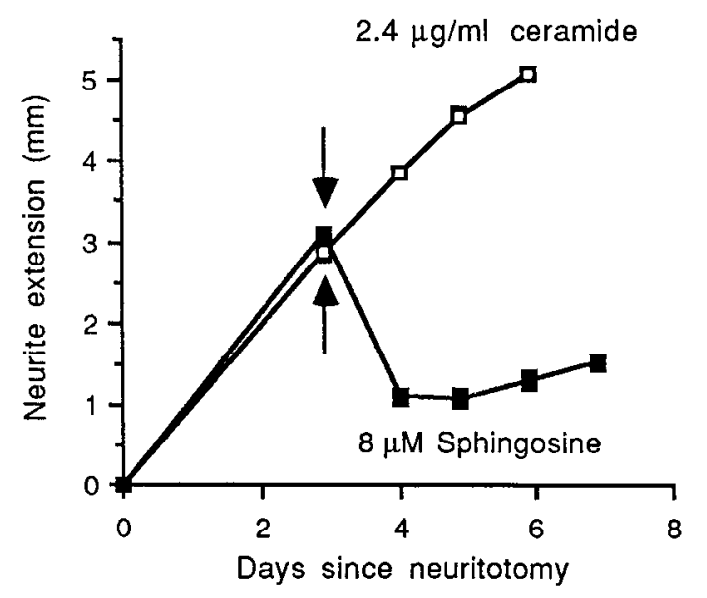

C

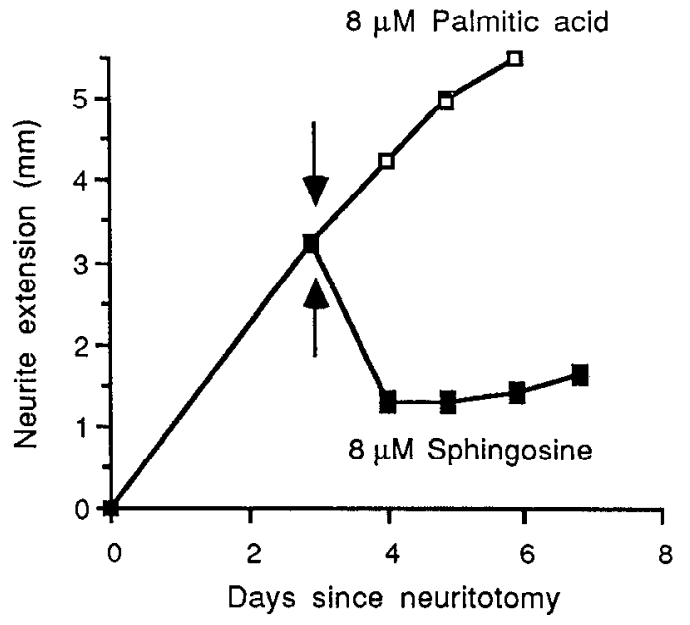

in 2 cultures neuritotomized on day 32.9 and $3.2 \mathrm{~d}$ later (arrows) given $8 \mu \mathrm{M}$ sphingosine in the right compartments (solid symbols) and $0.04 \%$ ethanol as a control in the left compartments (open symbols). $b$, Neurite extension in right and left compartments in 2 cultures neuritotomized on day 15.0 and $3.0 \mathrm{~d}$ later (arrows) given $8 \mu \mathrm{M}$ sphingosine in the right compartments (solid symbols) and $2.4 \mu \mathrm{g} / \mathrm{ml}$ ceramide in the left compartments (open symbols). c, Neurite extension in right and left compartments in 2 cultures neuritotomized on day 15.0 and $3.0 \mathrm{~d}$ later (arrows) given $8 \mu \mathrm{M}$ sphingosine in the right compartments (solid symbols) and $8 \mu \mathrm{M}$ palmitic acid in the left compartments (open symbols). 

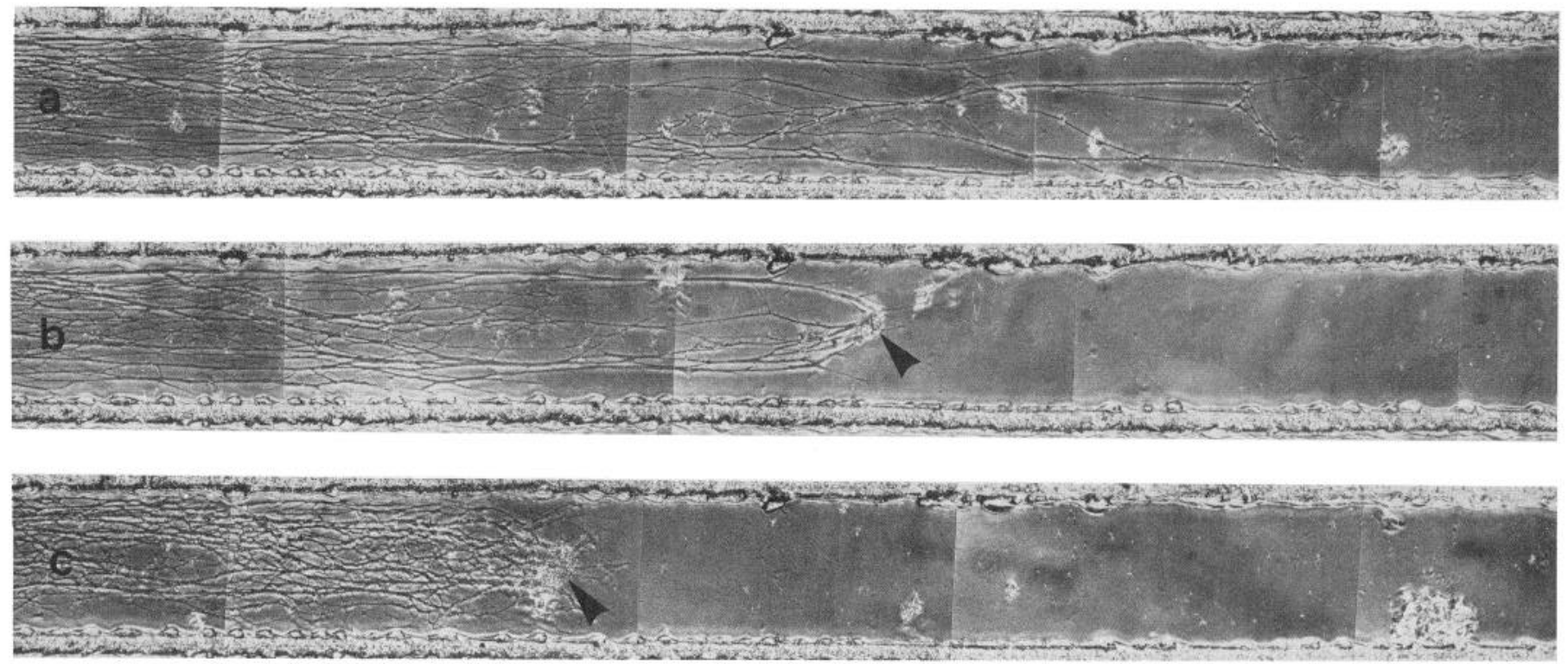

Figure 3. Response of neurites to sphingosine. $a$, Phase-contrast photomicrographs of neurites on a single track on day 25.0 in culture, $3.0 \mathrm{~d}$ after neuritotomy. The track width is $200 \mu \mathrm{m}$. The neurites extended $4.83 \mathrm{~mm}$, and only the distal $2.5 \mathrm{~mm}$ is shown. The neurites were then exposed to $8 \mu \mathrm{M}$ sphingosine. $b$, Photomicrographs taken $2.75 \mathrm{hr}$ after sphingosine addition. The neurites had regressed $0.95 \mathrm{~mm}$ from their original position and show a looplike ending (arrowhead). $c$, Photomicrographs taken $23 \mathrm{hr}$ after sphingosine addition. The neurites had regressed $1.34 \mathrm{~mm}$ from their original position in $a$, forming a ball-like ending (arrowhead). The total length of extension that remained in $c$ was 3.49 mm.

ments given either ceramide or palmitic acid extended at about the same rates as before treatment (Fig. $2 b, c)$. Also, these experiments showed again that neurite growth partially recovered within several days after sphingosine treatment.

In order to determine in detail how the distal neurites regressed in response to sphingosine, observations were made using time-lapse videorecording as well as photomicrography at time points shorter than $1 \mathrm{~d}$. Figures $3 a$ and $4 a$ show the typical appearance of neurites before sphingosine addition. Timelapse videorecordings of the distal ends of neurites showed that the effects of sphingosine were evident as early as $1-2 \mathrm{hr}$ after addition. Frequently, the first detectable event was the withdrawal of growth cones. The fibers leading to them shortened and thickened as they withdrew, and sometimes as the neurites withdrew, very fine fibers were drawn out from the neurite endings to the sites of original attachment. Eventually, as more fibers withdrew, they tended to coalesce into a looplike structure (Figs. $3 b$, arrowhead; $4 b$ ), which had numerous fine filaments projecting distally from it (Fig. $4 b$, arrowhead). Ultimately this looplike structure retracted further, forming a ball-like ending (Fig. $3 c$, arrowhead).

Because in the above experiments neurites recovered and began to reextend during several days of treatment with sphingosine, an experiment was performed to determine if sphingosine lost its effectiveness during exposure of the neurites. Three center-plated cultures were neuritotomized on day 8.0 and allowed to regenerate with unaltered conditions for $3.8 \mathrm{~d}$. Neurites in right compartments had extended $2.68 \pm 0.15 \mathrm{~mm}(N=48)$. Right compartments were then given medium containing $10 \mu \mathrm{M}$ sphingosine transferred from the side compartments of 3 other cultures where the medium had been in place for the preceding $3.8 \mathrm{~d}$. On day 1.1 after the medium transfer, neurite extension in right compartments was little changed at $2.65 \pm 0.17 \mathrm{~mm}$. Thus, previously used medium did not cause neurite retraction or degeneration, suggesting that sphingosine lost some of its effectiveness during several days exposure of neurites. Therefore, the partial recovery of neurite extension during several days of sphingosine treatment shown in the above experiments probably does not represent accommodation of neurites to sphingosine, but rather indicates recovery of neurite extension as the effectiveness of sphingosine was lost.

\section{Sphingosine has little or no effect when applied to cell bodies and proximal neurites}

It is noteworthy that in some experiments the more proximal neurites within side compartments given sphingosine were not obviously affected while the distal fibers peeled back (Fig. $3 c$ ). This could indicate that the mechanisms involved in the sphingosine effect are localized to the distal regions of the neurites. In order to test this, center-plated cultures were neuritotomized on day 8.0 , by which time neurites on many tracks had extended beyond $5 \mathrm{~mm}$. Because neurites had already reached the ends of some tracks, a quantitative assessment of neurite extension before treatment was not possible. Two of the cultures were then given $8 \mu \mathrm{M}$ sphingosine in only the center compartments containing the cell bodies and proximal neurites, while 2 others were given $8 \mu \mathrm{M}$ sphingosine in only the right compartments. All compartments not given sphingosine received the ethanol vehicle. In cultures treated with sphingosine in the center compartments, not a single track out of a combined left and right total of 70 tracks showed any shortening of neurite extension $0.8 \mathrm{~d}$ after sphingosine administration. On the contrary, neurite extension had continued, and cell bodies and proximal neurites directly exposed to sphingosine appeared normal. The lack of effect of sphingosine when applied to cell bodies and proximal neurites is shown in Figure $5 b$, which consists of photomicrographs of a culture that had been given $8 \mu \mathrm{M}$ sphingosine in the center compartment $0.9 \mathrm{~d}$ previously.

In contrast, cultures given sphingosine in the right compartments displayed massive degeneration localized to the right 

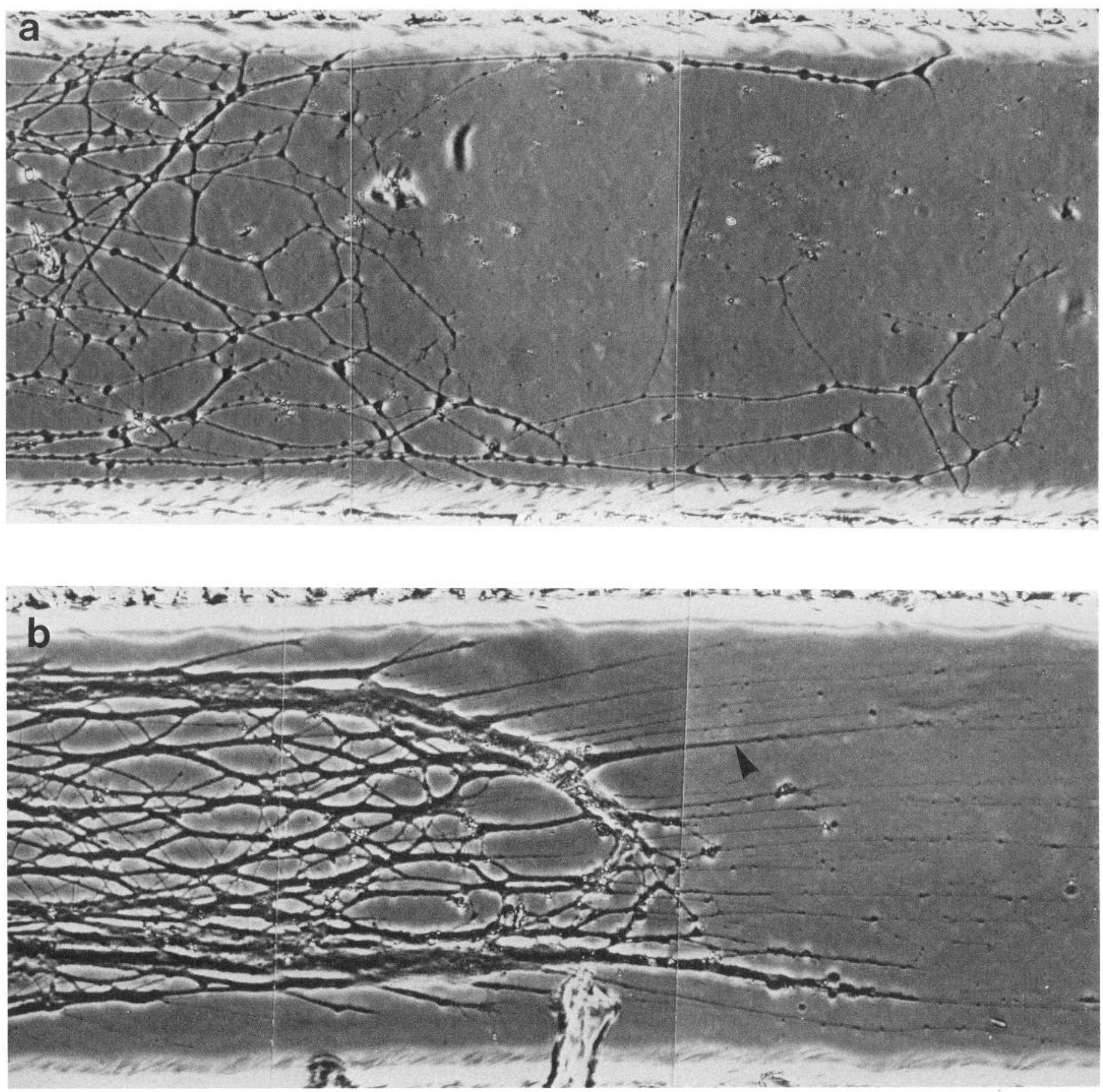

Figure 4. High-resolution phase-contrast photomicrographs of distal neurite endings given sphingosine. $a$, Neurite endings on a single track 4 min after sphingosine addition and before any effects became evident. $b$, Neurite endings on the same track $5 \mathrm{hr}$ later. The neurites had peeled back to form a looplike structure in a more proximal position on the track. Fine filaments extended distally from this structure (arrowhead). This culture had been neuritotomized on day 13.0, and the experiment was performed after $1.7 \mathrm{~d}$ of regeneration. The track width is $235 \mu \mathrm{m}$.

compartments. The neurites remaining $0.8 \mathrm{~d}$ after sphingosine addition averaged only $0.37 \pm 0.03 \mathrm{~mm}(N=34)$, a loss exceeding $4.5 \mathrm{~mm}$. However, the appearance of the degeneration was different from that described above. Instead of a peeling back of the distal neurites, leaving behind a track surface relatively free of debris, much debris remained behind, indicating that many of the neurites had disintegrated in place. Figure $5 a$ shows debris from neurites that degenerated in response to 8 $\mu \mathrm{M}$ sphingosine given $0.9 \mathrm{~d}$ previous to taking the photomicro- graph. The distal end of the debris on this track formed a looping structure (Fig. 5a, arrowhead), which was also seen in many other tracks. Apparently, the peeling back, such as was evident in Figure $3 b$, was in progress when the neurites disintegrated in place. It is not apparent why sphingosine produced predominantly a peeling back when applied to distal neurites in some experiments, but caused a massive disintegration of neurites in others.

To test further the specificity of sphingosine-induced destruc- 
a
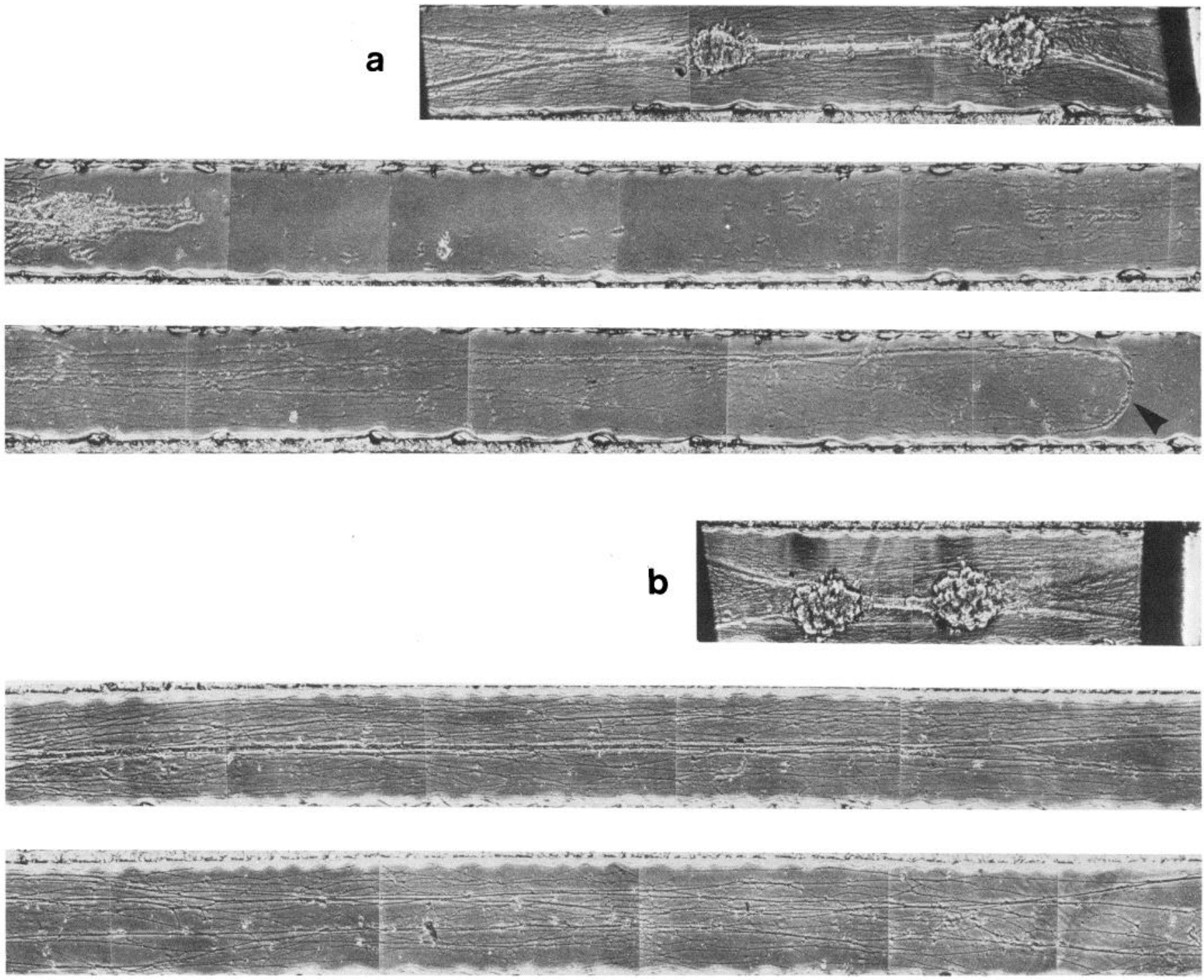

Figure 5. Phase-contrast photomicrographs comparing the effect of sphingosine on distal neurites versus on cell bodies and proximal neurites. Cultures maintained since day 7.0 with NGF in the side compartments only were neuritotomized on day 8.0 and given NGF in all compartments. Then, on day 12.1, the cultures were given $8 \mu \mathrm{M}$ sphingosine in different compartments. $a$, A continuous sequence of photomicrographs taken on day 13.0 of a culture given sphingosine in the right compartment only. The top panel shows cell bodies in the center compartment and edges of the silicone grease barriers on each side. The middle and bottom panels show the remains of neurites in the right compartment. It is clear that debris was left behind and that the neurites degenerated nearly completely back to the barrier. The end of the debris shows a rounded configuration characteristic of the initial stages of peeling back (arrowhead; cf. with Figs. $3 b, 4 b$ ). The width of this track is $202 \mu \mathrm{m}$. $b$, Continuous photomicrographs taken on day 13.0 of a culture given sphingosine in the center compartment. The layout is as in $a$. There was no obvious effect. The width of this track is $214 \mu \mathrm{m}$.

tion for distal neurites, an experiment was performed using a higher concentration of sphingosine. Center-plated cultures were neuritotomized on day 24.0 and allowed to regenerate for 3.0 $\mathrm{d}$, and then 2 cultures were given $100 \mu \mathrm{M}$ sphingosine in the center compartments only while 1 culture was given $100 \mu \mathrm{M}$ sphingosine in the right compartment and ethanol vehicle $(0.5 \%)$ in the left compartment. In the latter culture, neurites in the right compartment that had extended $2.79 \pm 0.16 \mathrm{~mm}(N=9)$ were virtually completely destroyed within $0.7 \mathrm{~d}$ after sphingosine administration. Short neurites were present on only 3 tracks. Neurites in the left compartment continued to elongate, extending from $2.92 \pm 0.32 \mathrm{~mm}$ to $3.52 \pm 0.31 \mathrm{~mm}$ during the $0.7-\mathrm{d}$ interval. In the cultures given $100 \mu \mathrm{M}$ sphingosine only in the center compartments, neurite extension in left and right compartments continued from $3.25 \pm 0.11 \mathrm{~mm}(N=34)$ before sphingosine administration to $4.13 \pm 0.11 \mathrm{~mm} 0.7 \mathrm{~d}$ after. Neurite extension also continued the second day after sphingosine addition, at which time neurites on many of the tracks were off scale for measurement. The neurites in the side compartments were completely normal in appearance, as were the cell bodies in the center compartments directly exposed to 100 $\mu \mathrm{M}$ sphingosine. However, many of the neurite bundles in the center compartments had acquired a granular appearance. Thus, $100 \mu \mathrm{M}$ sphingosine applied to cell bodies and proximal neurites had some effect, though this was not sufficient to cause their destruction.

Three experimental details of the above experiments need further consideration. First, when medium is changed in the 
center compartments of the cultures, a small amount of the original medium remains in the slot directly bathing the cell bodies and proximal neurites (see Fig. 1). Thus, any agent supplied in the new medium must diffuse to the cell bodies and proximal neurites to have an effect. While diffusion is presumably rapid relative to the time scale of the present results, the possibility does exist, though unlikely, that the comparatively slower rise of sphingosine concentration experienced by the cell bodies and proximal neurites might result in an attenuated effect compared to the immediate exposure experienced by distal neurites in the side compartments. To determine if an immediate exposure to a high level of sphingosine would affect cell bodies and proximal neurites, 2 center-plated cultures were neuritotomized on day 13.1 , and $1.8 \mathrm{~d}$ later the center compartments were given medium containing $100 \mu \mathrm{M}$ sphingosine. The slot was aspirated and refilled twice with sphingosine-containing medium to insure immediate exposure of the neurons to 100 $\mu \mathrm{M}$ sphingosine. Then, the outer perimeter was filled with medium as usual. In 1 of the cultures, some of the neurons were apparently mechanically damaged during the aspiration-refilling procedure because neurites on some tracks continued to extend during the next day while neurites on other tracks degenerated. In the other culture, neurites on all but 1 track in the left compartment and 1 track in the right compartment continucd to extend during the day after sphingosine administration. Mean neurite extension was $2.04 \pm 0.06 \mathrm{~mm}(N=36)$ before sphingosine treatment and $2.90 \pm 0.14 \mathrm{~mm} 1.1 \mathrm{~d}$ later, indicating that neurite growth had continued. Thus, immediate exposure to $100 \mu \mathrm{M}$ sphingosine did not destroy the cell bodies and proximal neurites, confirming the results of previous experiments.

Second, it was necessary to make certain that distal neurites would in fact degenerate within $1 \mathrm{~d}$ if the cell bodies and proximal neurites were destroyed. Two center-plated cultures were neuritotomized on day 8.0 and allowed to regenerate for $1.8 \mathrm{~d}$, at which time the cell bodies and proximal neurites were mechanically removed with sterile, distilled water delivered with a syringe (see Materials and Methods). The water was removed, and culture medium was added back to the center compartments. This had no immediate effect on the neurites in the left and right compartments, but $1 \mathrm{~d}$ later the neurites had virtually completely degenerated. This indicates that, had treatment of cell bodies and proximal neurites with sphingosine caused the destruction of proximal neurites, this would certainly have resulted in the degeneration of distal neurites during the period of observation. Because the distal neurites, on the contrary, continued to elongate, it can be concluded that sphingosine treatment did not cause the destruction of proximal neurites.

Third, the fact that medium supplied to cell bodies and proximal neurites in the center compartments contained $2.5 \%$ rat serum while the distal neurites in the side compartments were given medium without serum needs consideration. It is unlikely, but possible, that the presence of serum was responsible for the lack of effect of sphingosine on cell bodies and proximal neurites. For example, the presence of albumin could conceivably have reduced the concentration of sphingosine to which the neurons were actually exposed. While this cannot be ruled out in the above experiments, it was ruled out in experiments with leftplated cultures described in the next section.

In conclusion, the present work demonstrates that sphingosine, even at a 10 -fold greater concentration than that which caused severe degeneration of distal neurites, did not destroy cell bodies and proximal neurites.
Sphingosine-induced degeneration is specific for distal neurites

The above results suggest that the effect of sphingosine may be specific for distal neurites and leave proximal neurites unaffected. However, interpretation may be more complicated than is apparent at first sight because additional modes of action of sphingosine could have come into play when cell bodies were cxposcd to sphingosinc along with the proximal neurites. Therefore, in order to determine the effect of exposure of proximal neurites to sphingosine without exposure of the cell bodies, leftplated cultures, in which the cell bodies resided in the left compartments and neurites spanned the center compartments and entered the right compartments, were used (Fig. 1c). There were 2 cultures in each experimental treatment. NGF was provided only in the right compartments during the entire course of the experiment. On day 16.8 , neurites in the right compartments were neuritotomized and allowed to regenerate until day 21.0 . Next, neurite extension was measured, and the cultures were treated with 1 of 5 different distributions of $8 \mu \mathrm{M}$ sphingosine, following which neurite extension was measured again the next day (see Fig. 6a). Neurite regression was only observed when the neurites in the right compartments were directly exposed to sphingosine. Distal neurites in the right compartments continucd to elongate when the neurons were given sphingosine only in the left compartments containing the cell bodies or only in the center compartments containing the proximal neurites. Cell bodies and proximal neurites exposed to sphingosine showed no obvious deleterious effects.

Because only the distal neurites in the right compartments had been supplied with NGF during this experiment, it is possible that the simultaneous presence of NGF is required for sphingosine to cause neurite destruction. This possibility was checked with left-plated cultures. On day 14.9, neurites in the right compartments were removed, and NGF was then provided in the center and right compartments. Three days after neuritotomy, $8 \mu \mathrm{M}$ sphingosine was added to only proximal neurites in the center compartments in 4 cultures and only to the distal neurites in the right compartments in the 4 remaining cultures. Again, distal neurites exposed to sphingosine were destroyed by the next day while proximal neurites exposed to sphingosine were not, and their distal ends continued to elongate (Fig. 6b). Thus, the ability to destroy proximal neurites was not conferred upon sphingosine by the presence of NGF.

In all of these experiments with left-plated cultures, the left and center compartments had been NGF deprived after 6-10 $d$ in culture in order to concentrate neurite growth in the right compartments. This maneuver raises the possibility that proximal neurites were rendered resistant to the sphingosine effects because they had been previously deprived of NGF. To test this, left-plated cultures were used that had NGF always supplied to all compartments, that is, immediately from the time of plating and throughout the course of the experiment. On day 14.8, neurites in the right compartments were neuritotomized and allowed to regenerate for $3.2 \mathrm{~d}$. In 4 cultures, medium containing sphingosine $(8 \mu \mathrm{M})$ was added to the right compartments, while medium containing ethanol vehicle was added to the center compartments. In 5 other cultures, the treatments were reversed, with right compartments receiving ethanol-containing medium and center compartments receiving sphingosine-containing medium. As in previous experiments, distal neurites exposed to sphingosine were destroyed while proximal neurites exposed to sphingosine were not, and their distal ends continued to elongate 
Figure 6. Neurite extension in right compartments of left-plated cultures under different distributions of $8 \mu \mathrm{M}$ sphingosine. Cell bodies were located in the left compartments; neurites spanned the center compartments and elongated into the right compartments. Neurites in right compartments were neuritotomized and allowed to regenerate for 3-4 d, neurite extension was measured, and then $8 \mu \mathrm{M}$ sphingosine was added to the compartments indicated. Neurites were measured again the next day, and each pair of bars represents neurite extension before (open bar) and $1 \mathrm{~d}$ after (solid bar) sphingosine addition. Error hars are the SFM. $a$ shows results from an experiment in which NGF was supplied only in the right compartments. Cultures were $16.8 \mathrm{~d}$ old at the start of the experiment, 2 cultures were used for each treatment, and $N=$ 38 or 39 tracks for each group. Treatment consisted of 5 different distributions among the compartments of $8 \mu \mathrm{M}$ sphingosine, as indicated below each pair of bars. $b$ shows results in which NGF was supplied in the center and right compartments since neuritotomy. Cultures were $14.9 \mathrm{~d}$ old at the start of the experiment, and 4 cultures were used for each treatment. Treatment consisted of supplying $8 \mu \mathrm{M}$ sphingosine to either the center or right compartments as indicated, and $N=69$ and 70 tracks, respectively, for each group. $c$ shows results with cultures in which NGF was always supplied in all compartments from day 0 onward. Cultures were 14.8 d old at the start of the experiment. Treatment consisted of supplying $8 \mu \mathrm{M}$ sphingosine either to the center or right compartments as indicated, and 5 and 4 cultures, respectively, were used. $N=$ 80 and 71 tracks, respectively.
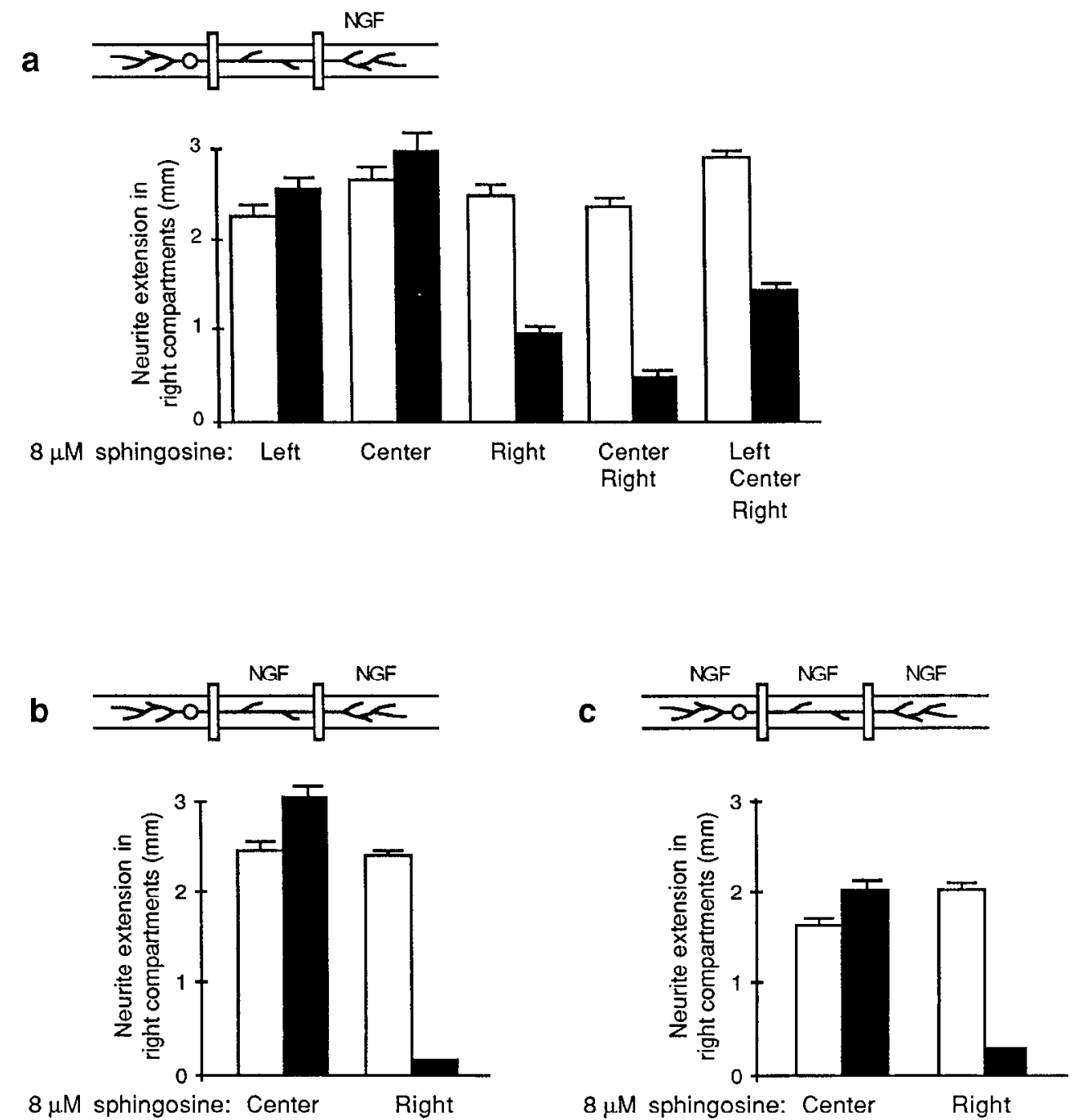

(Fig. 6c). Except for the presence or absence of sphingosine and ethanol vehicle, identical medium was always provided to proximal and distal neurites in all these cultures. The only difference was that the neurites residing in the center compartments were proximal while those in the right compartments were distal with respect to the cell bodies. Thus, the sphingosine-induced destruction is selective for distal neurites. Whether this sensitivity to sphingosine is conferred upon distal neurites because of their distal relationship to the cell body per se or because they were recently regenerated and therefore constituted new growth cannot be determined from these experiments.

\section{Staurosporine does not cause neurite retraction or degeneration}

In order to determine if staurosporine, another blocker of PKC, has effects on neurites similar to sphingosine, a center-plated culture was neuritotomized on day 9.9 , allowed to regenerate $3.1 \mathrm{~d}$, and then given $3 \mathrm{~nm}$ staurosporine in the right compartment. The left compartment was given equivalent DMSO ve- hicle as a control $\left(1.5 \times 10^{-4 \%}\right)$. Measurements $0.8 \mathrm{~d}$ after staurosporine addition showed that the neurites had extended about $0.60 \mathrm{~mm}$ in the left compartments and $0.64 \mathrm{~mm}$ in the right compartments since treatment. Neurite extension had increased on 18 of 20 tracks in the left compartment and on all 20 tracks in the right compartment, and there were no signs of degeneration.

To determine if a higher concentration of staurosporine might destroy distal neurites, 2 center-plated cultures were neuritotomized on day 10.0, allowed to regenerate for $3.0 \mathrm{~d}$, and were then given $100 \mathrm{nM}$ staurosporine in the right compartments and equivalent DMSO $(0.5 \%)$ in the left compartments. Here, too, neurite measurements $0.8 \mathrm{~d}$ after staurosporine addition showed that growth had continued in the left and right compartments, with mean neurite extension increasing by 0.69 and $0.87 \mathrm{~mm}$, respectively. Of the 37 tracks, neurite extension increased on all but 1 in the left compartments and on all but 1 in the right compartments. There were no signs of degeneration. On day 1.8 after staurosporine addition, mean neurite extension had in- 
creased by a total of $1.51 \mathrm{~mm}$ in the left compartments and 1.79 $\mathrm{mm}$ in the right compartments.

Even at extremely high concentrations, staurosporine did not cause neurite degeneration. Six center-plated cultures were neuritotomized on day 6.0 and allowed to regenerate until day 9.0 with unaltered conditions. Then, one culture served as control with no staurosporine (and no DMSO) in either compartment, and the other cultures were given staurosporine at concentrations of $10 \mathrm{~nm}, 50 \mathrm{~nm}, 100 \mathrm{~nm}, 500 \mathrm{~nm}$, or $1 \mu \mathrm{M}$. Both left and right compartments in each culture were given the same concentration. The concentrations were achieved by serial dilution of the $1-\mu \mathrm{M}$ staurosporine-containing medium that had a DMSO concentration of $0.05 \%$. The cultures were examined $0.8 \mathrm{~d}$ later. Figure 7 shows that neurite extension was slowed by staurosporine concentrations of $100 \mathrm{~nm}$ and above, but no concentration of staurosporine caused neurite degeneration.

A final cxpcriment confirmed that high concentrations of staurosporine depressed neurite growth. One culture was neuritotomized on day 13.0 and allowed to regenerate for $1.8 \mathrm{~d}$ with unaltered conditions. The right compartment was then given 1 $\mu \mathrm{M}$ staurosporine, and the left compartment was given equivalent DMSO $(0.05 \%)$. Before treatment, neurites in the right compartment extended $2.26 \pm 0.08 \mathrm{~mm}(N=18)$, and $1.0 \mathrm{~d}$ after treatment, extension was virtually unchanged at $2.28 \pm$ $0.08 \mathrm{~mm}$. Neurite extension on the majority of individual tracks was little changed. In the left compartment, however, mean neurite extension had increased by $1.06 \mathrm{~mm}$. Thus, the DMSO itself did not appear to depress the rate of neurite extension below what is usually observed.

\section{PMA causes retraction of neurites, from which they recover}

In order to investigate the possible involvement of $\mathrm{PKC}$ in neurite growth from another perspective, an activator/downregulator of PKC, PMA, was applied to regenerated neurites. Two center-plated cultures were neuritotomized on day 8.0 and allowed to regenerate $4.0 \mathrm{~d}$ with unaltered conditions. Neurites averaged $4.17( \pm 0.21) \mathrm{mm}$ in the left compartments and 4.38 $( \pm 0.19) \mathrm{mm}$ in the right compartments at this time. Then, left compartments were given DMSO vehicle $(0.1 \%)$, and right compartments were given $2 \mu \mathrm{M}$ PMA. Neurites in the control compartments were unaffected, elongating to $4.75( \pm 0.22) \mathrm{mm}$ at $0.8 \mathrm{~d}$ after treatment. However, neurite extension in compartments given PMA decreased to $3.65( \pm 0.30) \mathrm{mm}$ at $0.8 \mathrm{~d}$ after treatment, an average loss of $0.73 \mathrm{~mm}$. This was a smaller loss, leaving behind a much greater length of neurites, than was typically seen with $8 \mu \mathrm{M}$ sphingosine. In fact, there was considerable variability between tracks, with 20 of the 29 tracks showing a decrease in neurite extension and 9 tracks actually showing an increase in neurite extension. Even though PMA was not removed, neurites in the right compartments recovered from this effect; by $1.8 \mathrm{~d}$ post-PMA addition, they had reextended an average of $0.43 \mathrm{~mm}$ from the value at $0.8 \mathrm{~d}$ post-PMA addition, with every track showing an increase.

\section{Chronic PMA treatment slows neurite growth but does not block the effect of sphingosine}

Because neurites recovered and began to reextend during treatment with $2 \mu \mathrm{M}$ PMA, it was possible to regenerate neurites chronically given PMA and test the effect of sphingosine in the presence of PMA. Because chronic PMA downregulates PKC in cultured rat sympathetic neurons (see introductory remarks and Discussion), the sphingosine-induced degeneration should

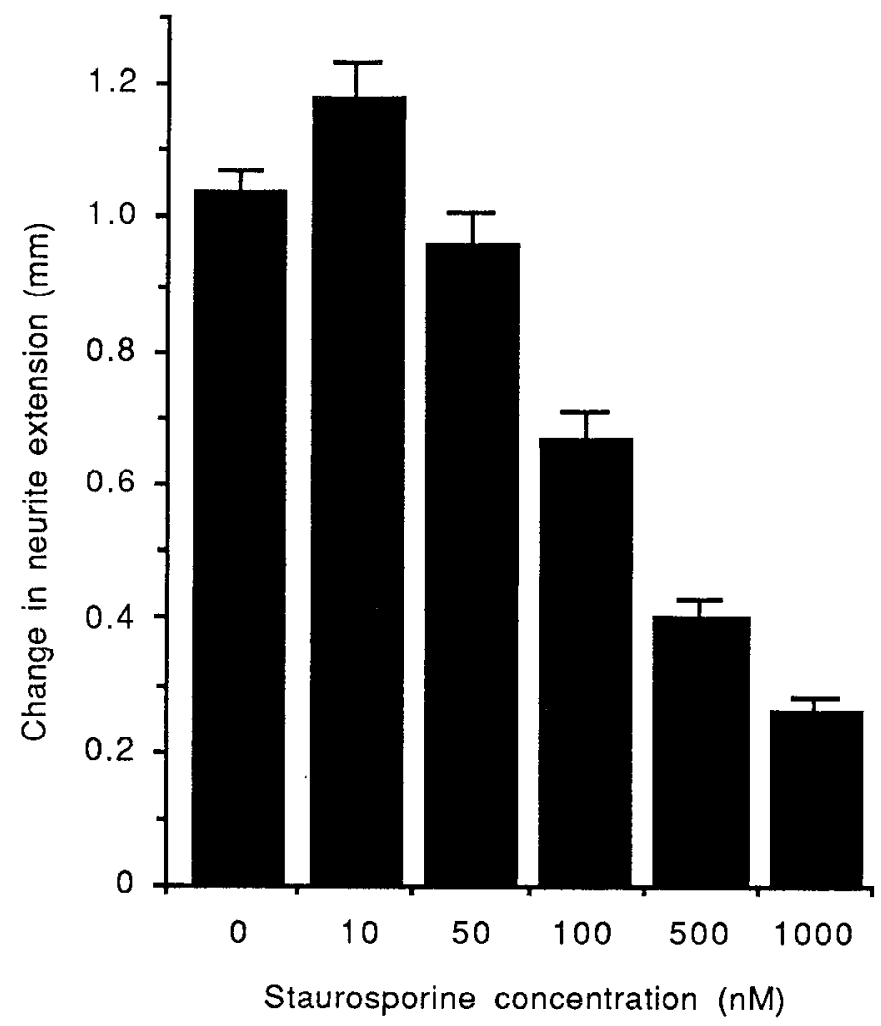

Figure 7. Combined neurite extension in left and right compartments of cultures treated with staurosporine in left and right compartments. Six center-plated cultures were neuritotomized on day 6.0 and allowed to regenerate until day 9.0 with unaltered conditions. Then, one culture served as control with no staurosporine (and no DMSO) in either compartment, and the other cultures were given staurosporine at concentrations of $10 \mathrm{nM}, 50 \mathrm{nM}, 100 \mathrm{nM}, 500 \mathrm{nM}$, or $1 \mu \mathrm{M}$. Both left and right compartments in each culture were given the same concentration, and the left and right measurements were pooled. Plotted is the change in neurite extension in each culture $1 \mathrm{~d}$ after staurosporine addition calculated by subtracting neurite extension on day 8.9 from neurite extension on day 9.8. Error bars are the SEM, and $N$ was 34-36 tracks for each culture.

be blocked by PMA if PKC is involved in the mechanism of the sphingosine effect. Four cultures with neurons plated in center compartments were neuritotomized on day 15.0 and given $2 \mu \mathrm{M}$ PMA in the right compartments and DMSO vehicle $(0.1 \%)$ in the left compartments. Measurements $2.9 \mathrm{~d}$ later showed that PMA-treated neurites had extended $46 \%$ of the distance extended by control neurites (see Fig. $8 a$ ). To insure potency of the PMA, $1.2 \mathrm{hr}$ after these measurements were completed, the medium in the side compartments was replaced with fresh medium, still with DMSO left and $2 \mu \mathrm{M}$ PMA right. Neurites were measured again, $2.3 \mathrm{hr}$ after the medium change, and mean neurite extension had increased by about $60 \mu \mathrm{m}$ in the PMA-treated compartments during the $3.5 \mathrm{hr}$ since the last measurements. Thus, the fresh PMA did not itself cause neurites to regress. Immediately after these measurements were made, the medium in left and right compartments was changed again. Left compartments were given DMSO and $8 \mu \mathrm{M}$ sphingosine, and right compartments were given $2 \mu \mathrm{M}$ PMA and $8 \mu \mathrm{M}$ sphingosine. Measurements were made again $0.7 \mathrm{~d}$ after sphingosine treatment, and Figure $8 a$ shows that neurites on both sides had regressed. Thus, chronic treatment with PMA did not block the ability of sphingosine to cause neurite regression.

This result was confirmed in an experiment with a different 


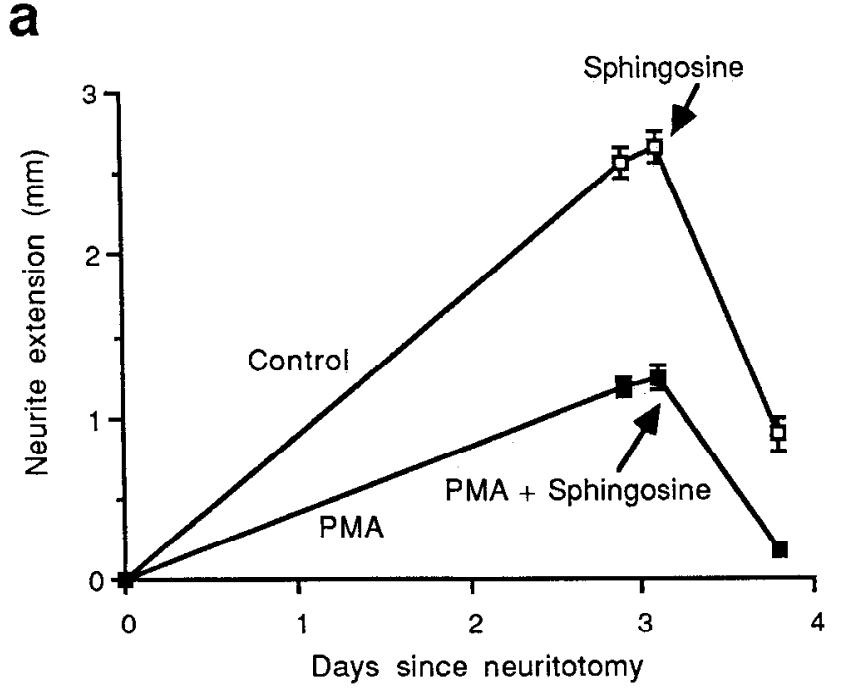

b

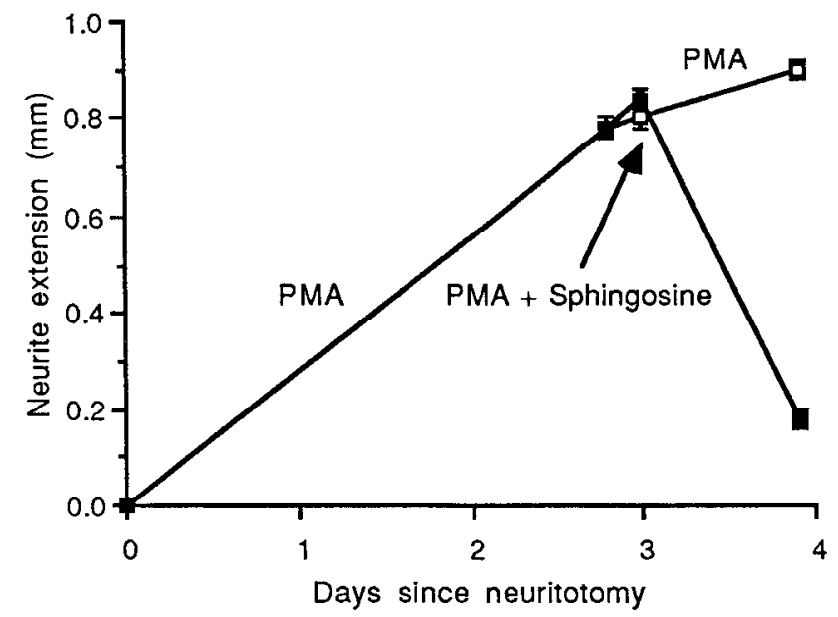

Figure 8. Effect of sphingosine on cultures pretreated with PMA. Error bars are the SEM and, in some cases, are included within the symbols. $a$, Neurite extension in 4 cultures neuritotomized on day 15.0 and given culture medium containing DMSO vehicle $(0.1 \%)$ in the left compartments (open symbols) and $2 \mu \mathrm{M}$ PMA in the right compartments (solid symbols). Neurites were measured $2.9 \mathrm{~d}$ later, given fresh medium again with DMSO left and $2 \mu \mathrm{M}$ PMA right, measured again $2.3 \mathrm{hr}$ after the medium change, and then given medium with DMSO and $8 \mu \mathrm{M}$ sphingosine left and with $2 \mu \mathrm{M}$ PMA and $8 \mu \mathrm{M}$ sphingosine right. $b$, Neurite extension in left (open symbols) and right compartments (solid symbols) in 3 cultures neuritotomized on day 17.0 and given $2 \mu \mathrm{M}$ PMA in all compartments. Neurites were measured $2.8 \mathrm{~d}$ later, and then the medium in the side compartments was replaced with fresh medium again containing $2 \mu \mathrm{M}$ PMA. The center compartment medium was also replaced with fresh medium containing $2 \mu \mathrm{M}$ PMA. About $4.5 \mathrm{hr}$ after the side compartment medium had been changed, the neurites were measured again, and then the left compartment medium was replaced with medium containing $2 \mu \mathrm{M}$ PMA and $0.05 \%$ ethanol, and the right compartment was given medium containing $2 \mu \mathrm{M}$ PMA and $10 \mu \mathrm{M}$ sphingosine.

design. Three cultures were neuritotomized on day 17.0 and given $2 \mu \mathrm{M}$ PMA in all compartments so as to downregulate PKC everywhere in the neurons. Neurites were measured $2.8 \mathrm{~d}$ later and had extended a similar distance to neurites locally supplied with PMA in the previous experiment. Thus, the PMA treatment again dcpressed the rate of neurite extension. The medium in the side and center compartments was replaced with fresh medium containing $2 \mu \mathrm{M}$ PMA, and, as in the above experiment, neurites continued to advance. Then (3.1 d since neuritotomy), the medium in the left compartments was replaced with medium containing $2 \mu \mathrm{M}$ PMA and $0.05 \%$ ethanol, and the medium in the right compartments was replaced with medium containing $2 \mu \mathrm{M}$ PMA and $10 \mu \mathrm{M}$ sphingosine. The next day, the neurites in the left compartments given PMA had continued to extend, while in the right compartments neurites given PMA and sphingosine had regressed nearly completely (Fig. $8 b$ ). This shows again that chronic PMA treatment did not block the effect of sphingosine.

\section{Anti-NGF treatment does not cause immediate retraction or degeneration of distal neurites}

To investigate whether the sphingosine-induced destruction of distal neurites might arise solely by blocking of the action of NGF, 3 center-plated cultures neuritotomized on day 10.8 were allowed to regenerate for $3.2 \mathrm{~d}$ with NGF supplied in left and right compartments. Then, the left compartments were given NGF-supplied medium, but NGF was removed from the right compartments by supplying medium containing $24 \mathrm{nM}$ antiNGF IgG instead of NGF. Neurites in the NGF-supplied left compartments continued to extend and were off scale on many tracks within $1 \mathrm{~d}$ after the medium change. Neurite extension in the right compartments was arrested in response to the removal of NGF; the mean value was $4.45 \pm 0.06 \mathrm{~mm}(N=54)$ at the time of the medium change and $1.0 \mathrm{~d}$ later was unchanged at $4.46 \pm 0.07 \mathrm{~mm}$. At 2.1 and $3.0 \mathrm{~d}$ after the medium change, mean neurite extension was, respectively, $4.46 \pm 0.08 \mathrm{~mm}$ and $4.49 \pm 0.07 \mathrm{~mm}$. Thus, while removal of NGF arrested neurite extension, unlike sphingosine treatment, it did not result in retraction or degeneration. It is therefore unlikely that a hypothetical block of NGF action by sphingosine could have been responsible for the sphingosine-induced retraction and degeneration of distal neurites.

\section{Discussion}

Role of PKC activity in the control of neurite growth in rat sympathetic neurons

The present results suggest that PKC activity is not necessary for neurite growth in cultured rat sympathetic neurons. Although sphingosine, a well-known blocker of PKC activity (Hannun and Bell, 1989), caused the specific retraction and degeneration of distal neurites, staurosporine, which blocks PKC in rat brain with an $\mathrm{IC}_{\mathrm{so}}$ of $2.7 \mathrm{nM}$ (Tamaoki et al., 1986), did not cause degeneration when locally applied to distal neurites, either at $3 \mathrm{nM}$ or at higher concentrations up to $1 \mu \mathrm{M}$. Staurosporine in the nanomolar range has been reported to have complex effects on neurite growth in PC12 cells (Hashimoto and Hagino, 1989), but in the present study staurosporine concentrations of up to $50 \mathrm{~nm}$ had little effect on neurite extension in sympathetic neurons. At concentrations ranging from $100 \mathrm{nM}$ to $1 \mu \mathrm{M}$, staurosporine reduced neurite extension in a dose-dependent fashion. It is unknown why higher concentrations than required for effects in PC12 cells were needed to show an effect in sympathetic neurons, but the present results nonetheless suggest that neurite growth can occur in PKC-inhibited neurons.

Further evidence that PKC activity is not required for neurite growth was provided by cultures treated with phorbol ester. It has been shown that $24 \mathrm{hr}$ exposure to $1 \mu \mathrm{M}$ PMA virtually 
completely downregulated PKC activity in sympathetic neurons from superior cervical ganglia of newborn rats cultured under very similar conditions as used here (Matthies et al., 1987). The present results show that neurites exposed to $2 \mu \mathrm{M}$ PMA continued to extend for a 3-d observation period at about half the control rate. However, because there are several isozymes of PKC (Nishizuka, 1988), 2 of which display different time courses of downregulation in response to PMA (Huang et al., 1989), the question arises as to whether all isozymes had been downregulated in sympathetic neurons. However, the reported time required for downregulation in the isozymes tested never approached $24 \mathrm{hr}$; in RBL-2H3 cells, type II kinase was completely downregulated after about $30 \mathrm{~min}$ exposure to $20 \mathrm{nM}$ PMA, while complete downregulation of type III kinase required about $2 \mathrm{hr}$ (Huang et al., 1989). Second, in order to measure downregulation of PKC activity in sympathetic neurons, Matthies et al. (1987) used phosphorylation of histone $\mathrm{H} 1$, which has been shown to be phosphorylated by types I, II, and III PKC isozymes (Huang et al., 1988; for review, see Parker et al., 1989). The observation of Matthies et al. (1987) that phosphorylation of histone $\mathrm{H} 1$ was virtually abolished by 24-hr treatment of rat sympathetic neurons with PMA would therefore appear to be a good indication of downregulation of PKC activity.

Thus, the present result that neurite growth occurred in the presence of PMA suggests that neurite growth in rat sympathetic neurons does not require the activity of PKC. However, the observed reduction of extension rate also suggests that $\mathrm{PKC}$ may play a role in modulating neurite growth. In contrast to the present results, 24-hr pretreatment of PC12 cells with $1 \mu \mathrm{M}$ PMA had no effect on neurite sprouting in response to NGF (Reinhold and Neet, 1989). The present work suggests that sustained neurite growth in sympathetic neurons may be controlled by different mechanisms than those that control initial sprouting in PC12 cells.

\section{The retraction and degeneration of distal neurites in response to sphingosine is not mediated by inhibition of $P K C$}

If the sphingosine-induced retraction and degeneration of distal neurites is mediated by inhibition of PKC, then inhibition and downregulation of PKC by other means should produce similar effects. However, neither staurosporine nor long-term PMA treatment caused neurite retraction and degeneration. Furthermore, pretreatment of neurons for $3 \mathrm{~d}$ with $2 \mu \mathrm{M}$ PMA, which presumably eliminated PKC activity, did not prevent sphingosine from destroying distal neurites. This strongly suggests that the sphingosine-induced retraction and degeneration of distal sympathetic neurites are not mediated by PKC. These results with sympathetic neurons are consistent with the suggestion that inhibition of neurite sprouting in PC12 cells by sphingosine is not mediated by PKC (Reinhold and Neet, 1989).

\section{Sphingosine-induced retraction and degeneration does not arise through block of NGF action}

The possibility that the retraction and degeneration of distal neurites in response to sphingosine resulted from block of the ncuritc survival-promoting action of NGF is unlikely, because removal of NGF from distal neurites, accomplished by replacing NGF-containing medium with medium supplied instead with $24 \mathrm{nM}$ affinity-purified anti-NGF IgG, only stopped the growth of neurites; the existing neurites did not degenerate within $3 \mathrm{~d}$ of observation. This is consistent with earlier work (Campenot, 1982b) showing that neurites stopped extending and then de- generated slowly over a period of 2 weeks following NGF withdrawal. Thus, block of NGF action is unlikely to be the mechanism by which distal neurites are destroyed within $24 \mathrm{hr}$ after treatment with sphingosine.

\section{Biological significance of sphingosine-induced retraction and degeneration of distal neurites}

The retraction and degencration of distal ncurites observed in response to 1-10 $\mu \mathrm{M}$ sphingosine were not general toxic effects hecause even $100 \mu \mathrm{M}$ sphingosine was not toxic to the cells or proximal neurites. This is consistent with the observation that neurite sprouting in PC12 cells was reversibly inhibited by $80 \%$ during $2 \mathrm{~d}$ treatment with $10 \mu \mathrm{M}$ sphingosine, with most of the inhibited neurons sprouting neurites within $1 \mathrm{~d}$ after sphingosine withdrawal (Hall et al., 1988). Half-maximal inhibition of sprouting was reported to be at 2.5-5 $\mu \mathrm{M}$ sphingosine. Thus, in sympathetic neurons and in PC12 cells, sphingosine in the same concentration range has specific effects on neurites without general toxicity to the cells.

In some experimental systems, sphingosine or related compounds have been reported to kill cells: treatment with $6 \mu \mathrm{M}$ sphinganine killed cultured Chinese hamster ovary cells within $24 \mathrm{hr}$, but 1 or $2 \mu \mathrm{M}$ sphinganine had no deleterious effect on cell survival (Mcrrill, 1983); $10 \mu \mathrm{M}$ sphingosine has been reported to kill cultured cerebellar granule cells in just $2 \mathrm{hr}$ (Manev et al., 1990). Clearly, no such effect occurred in sympathetic neurons in the present study nor in work with PC12 cells (Hall et al., 1988).

The specificity of the sphingosine-induced retraction/degeneration for distal neurites has interesting implications. Sphingosine is an amphiphilic lipid that readily partitions into the plasma membrane (see Hannun and Bell, 1989), so it is unlikely to be able to enter membranes of distal neurites but not those of proximal neurites and cell bodies. Thus, the selectivity of distal neurites for degeneration probably does not result from selective access to sphingosine. In light of this, the present results may indicate that elements of the mechanism of sphingosineinduced retraction/degeneration are either localized to distal neurites or are selectively functional in distal neurites.

The actual mechanism of sphingosine-induced retraction/degeneration of distal neurites is unknown, but some possibilities are worth discussing. The initial interest in PKC stemmed partly from its role in the inositol phosphoglyceride signaling pathway (see Berridge, 1987; Berridge and Irvine, 1989). In this pathway, the binding of a ligand to a receptor on the cell surface results in the activation of phospholipase $\mathrm{C}$, which cleaves inositol phosphoglycerides in the membrane into inositol trisphosphate and diacylglycerol. These 2 products produce a dual signal: inositol trisphosphate, which is released into the cytoplasm, where it causes the release of $\mathrm{Ca}^{2+}$ from intracellular stores to give a cytoplasmic $\mathrm{Ca}^{2+}$ signal, and diacylglycerol, which remains in the plasma membrane, where it produces a PKC signal by binding to and activating the enzyme.

However, it seems unlikely that sphingosine caused the observed retraction/degeneration of neurites by interfering with the inositol phosphoglyceride signaling pathway for the following reasons: first, the most likely site of action for sphingosine to block this pathway is by blocking the activation of PKC, but the present results suggest that sphingosine does not produce its effects this way and that blocking of PKC by other means does not cause retraction or degeneration. Also, the activation of the inositol phosphoglyceride pathway is unlikely to be required for 
neurite maintenance because the pathway is believed to produce brief signals, not long-term signals (see review by Löffelholz, 1989). It is hard to see how a brief signal could support a longterm phenomenon such as neurite survival.

Another possible mechanism is suggested by a recent report that sphingosine activates phospholipase $\mathrm{D}$ in neural-derived NG108-15 cells (Lavie and Liscovitch, 1990). Phospholipase D is part of a newly emerging phospholipid signaling pathway (see review by Löffelholz, 1989) in which the phospholipase D hydrolysis of phosphotidylcholine produces free choline and phosphatidic acid. Phosphatidic acid can act as a signal on its own, and it can also be further metabolized to diacylglycerol, thereby activating PKC. It is conceivable that the maintenance of distal neurites might be regulated by activation of the phospholipase D signaling system, and that activation of this system by sphingosine could trigger neurite retraction/degeneration via phosphatidic acid or some action of diacylglycerol not involving PKC.

Another possible mechanism for effects of sphingosine is suggested by a recent report that sphingosine caused the release of $\mathrm{Ca}^{2+}$ from intracellular stores in cells of a smooth muscle line (Ghosh et al., 1990). Because it has been suggested that increases in cytosolic $\mathrm{Ca}^{2+}$ in growth cones can cause neurite growth to cease (Mattson and Kater, 1987), the possibility is raised that the sphingosine-induced retraction and degeneration of distal neurites observed in the present experments could have been mediated by a sphingosine-induced increase in cytoplasmic $\mathrm{Ca}^{2+}$.

A final aspect to be considered is that naturally occurring sphingosine or more complex sphingolipid breakdown products (lysosphingolipids) might themselves function as second messengers in cell regulation. Interest in this possibility has developed only recently, and little is known of the precise roles that sphingosine and lysosphingolipids may play (for revicw, scc Hannun and Bell, 1989). One speculative scenario would be that a membrane sphingolinid is metabolized in response to binding of an extracellular ligand, and the lysosphingolipid and/ or sphingosine resulting from this breakdown inhibits PKC and affects other phosphorylation systems as well. Because the present results suggest that $\mathrm{PKC}$ is not involved, effects on 1 or more of the other phosphorylation systems might then mediate the sphingosine-induced degeneration of distal neurites. This hypothesis in turn suggests the possibility of a ligand-activated, sphingolipid-mediated second-messenger system that can cause retraction and/or degeneration of nerve endings. It is conceivable that such a system could mediate regressive phenomena that naturally occur in the developing nervous system, such as synapse elimination and axon collateral elimination.

What ligands might function to activate this hypothetical system is unknown, but intercsting spcculations arisc from recent reports of membrane proteins that exert inhibitory influences on nerve growth. Two protein fractions have been isolated from rat CNS myelin that inhibit the growth of rat sympathetic neurites and dorsal root ganglion neurites (Caroni and Schwab, 1988a). Adsorption of CNS myelin or cultured oligodendrocytes with monoclonal antibodies against either fraction markedly improved neurite growth on these substrata (Caroni and Schwab, 1988 b). This suggests that these proteins are responsible for conferring upon the adult CNS its impermissiveness for nerve growth and regeneration. Similar phenomena have been described in other systems: growth cones of chick dorsal root ganglia collapse into nonmotile forms when presented with a suspension of embryonic chick brain membranes (Raper and
Kapfhammer, 1990) or with a glycoprotein fraction isolated from chick somites (Davies et al., 1990); and chick tectal membranes, especially those from the posterior tectum, cause the collapse of growth cones from temporal retina but not from nasal retina (Cox et al., 1990). It appears that membrane proteins that serve as neurite-growth-inhibitory ligands may be widespread within the nervous system and may play important roles in directing neurite growth and establishing appropriate connections. The inhibition of growth and especially the collapse of growth cones observed in these studies suggest a milder and/ or more localized version of the retraction and degeneration of distal nerve fibers observed in the present study in response to sphingosine. It is reasonable to hypothesize that collapse of growth cones in response to ligands bound to membranes of impermissive cells may be mediated by the local breakdown of growth cone sphingolipids.

The present work has possible implications for certain diseases involving the nervous system. Lysosphingolipids are known to accumulate in sphingolipidoses, a class of inherited disorders that includes Krabbe's disease, Gaucher's disease, and Tay-Sach's disease (see Volk and Schneck, 1975). Some of these involve accumulation of lysosphingolipids in the CNS and neuronal degeneration. The possibility has been raised that the mechanism of such dysfunctions may involve inhibition of PKC resulting from the accumulated lysosphingolipids (Hannun and Bell, 1987), but the present results suggest that nerve fiber degeneration could result from other effects of lysosphingolipid accumulation.

Finally, the present experiments suggest that there may be sphingolipid second-messenger systems that mediate normally occurring nerve growth inhibition and nerve fiber retraction and degeneration. Dysfunctions of such a mechanism of nerve ending degencration could underlic a large range of neurodegenerative disorders, and pharmacological manipulation of this system could be an approach for promoting nerve regeneration.

\section{References}

Ballester R, Rosen OM (1985) Fate of immunoprecipitable protein kinase $\mathrm{C}$ in $\mathrm{GH}_{3}$ cells treated with phorbol 12-myristate 13-acetate. J Biol Chem 260:15194-15199.

Berridge MJ (1987) Inositol trisphosphate and diacylglycerol: two interacting second messengers. Annu Rev Biochem 56:159-193.

Berridge MJ, Irvine RF (1989) Inositol phosphates and cell signalling. Nature 341:197-205.

Campenot RB (1979) Independent control of the local environment of somas and neurites. In: Methods in enzymology, Vol 28 (Jakoby WB, Pastan IH, eds), pp 302-307. New York: Academic.

Campenot RB (1982a) Development of sympathetic neurons in compartmentalized cultures: I. Local control of neurite growth by nerve growth factor. Dev Biol 93:1-12.

Campenot RB (1982b) Development of sympathetic neurons in compartmentalized cultures: II. Local control of neurite survival by nerve growth factor. Dev Biol 93:13-21.

Campenot RB (1987) Local promotion of neurite sprouting in cultured sympathetic neurons by nerve growth factor. Dev Brain Res 37:293301 .

Campenot KB, Draker DD (1989) Growth of sympathetic nerve fibers in culture does not require extracellular calcium. Neuron 3:733-743.

Caroni P, Schwab ME (1988a) Two membrane protein fractions from rat central myelin with inhibitory properties for neurite growth and fibroblast spreading. J Cell Biol 106:1281-1288.

Caroni P, Schwab ME (1988b) Antibody against myelin-associated inhibitor of neurite growth neutralizes nonpermissive substrate properties of CNS white matter. Neuron 1:85-96.

Cox EC, Müller B, Bonhoeffer F (1990) Axonal guidance in the chick visual system: posterior tectal membranes induce collapse of growth cones from the temporal retina. Neuron 2:31-37. 
Davies JA, Cook GMW, Stern CD, Keynes RJ (1990) Isolation from chick somites of a glycoprotein fraction that causes collapse of dorsal root ganglion growth cones. Neuron 2:11-20.

Faucher M, Gironès N, Hannun YA, Bell RM, Davis RJ (1988) Regulation of the epidermal growth factor receptor phosphorylation state by sphingosine in A431 human epidermoid carcinoma cells. J Biol Chem 263:5319-5327.

Ghosh TK, Bian J, Gill DL (1990) Intracellular calcium release mediated by sphingosine derivatives in cells. Science 248:1653-1656.

Greene LA, Tischler AS (1976) Establishment of a noradrenergic clonal line of rat adrenal pheochromocytoma cells which respond to nerve growth factor. Proc Natl Acad Sci USA 73:2424-2428.

Hall FL, Fernyhough P, Ishii DN, Vuillet PR (1988) Suppression of nerve growth factor-directed neurite outgrowth in $\mathrm{PCl} 2$ cells by sphingosine, an inhibitor of protein kinase C. J Biol Chem 263:44604466.

Hannun YA, Bell RM (1987) Lysosphingolipids inhibit protein kinase C: implications for the sphingolipidoses. Science 235:670-674.

Hannun YA, Bell RM (1989) Functions of sphingolipids and sphingolipid breakdown products in cellular regulation. Science 243:500507.

Hashimoto S, Hagino A (1989) Blockage of nerve growth factor action in $\mathrm{PC} 12 \mathrm{~h}$ cells by staurosporine, a potent protein kinase inhibitor. $\mathrm{J}$ Neurochem 53:1675-1685.

Hawrot E, Patterson PH (1979) Long-term cultures of dissociated sympathetic neurons. In: Methods in enzymology, Vol 28 (Jakoby WB, Pastan IH, eds), pp 574-584. New York: Academic.

Heasley LE, Johnson GL (1989) Regulation of protein kinase C by nerve growth factor, epidermal growth factor, and phorbol esters in PC12 pheochromocytoma cells. J Biol Chem 264:8646-8652.

Huang K-P, Huang FL, Nakabayashi H, Yoshida Y (1988) Biochemical characterization of rat brain protein kinase $\mathrm{C}$ isozymes. J Biol Chem 263:14839-14845.

Huang FL, Yoshida Y, Cunha-Melo JR, Beaven MA, Huang K-P (1989) Differential down-regulation of protcin kinase C isozymes. J Biol Chem 264:4238-4243.

Lavie Y, Liscovitch M (1990) Activation of phospholipase D by sphingoid bases in NG108-15 neural-derived cells. J Biol Chem 265:38683872 .

Löffelholz K (1989) Receptor regulation of choline phospholipid hydrolysis, a novel source of diacylglycerol and phosphatidic acid. Biochem Pharmacol 38:1543-1549.
Manev H, Favaron M, Vicini S, Guidotti A, Costa E (1990) Glutamate-induced neuronal death in primary cultures of cerebellar granule cells: protection by synthetic derivatives of endogenous sphingolipids. J Pharmacol Exp Ther 252:419-427.

Matthies HJG, Palfrey HC, Hirning LD, Miller RJ (1987) Down regulation of protein kinase $\mathrm{C}$ in neuronal cells: effects on neurotransmitter release. J Neurosci 7:1198-1206.

Mattson MP, Kater SB (1987) Calcium regulation of neurite elongation and growth cone motility. J Neurosci 7:4034-4043.

Merrill AH (1983) Characterization of serine palmitoyltransferase activity in Chinese hamster ovary cells. Biochim Biophys Acta 754: 284-291.

Nishizuka Y (1988) The molecular heterogeneity of protein kinase C and its implications for cellular regulation. Nature 334:661-665.

Parker PJ, Kour G, Marais RM, Mitchell F, Pears C, Schaap D, Stabel S, Webster C (1989) Protein kinase C-a family affair. Mol Cell Endocrinol 65:1-11.

Raper JA, Kapfhammer JP (1990) The enrichment of a neuronal growth cone collapsing activity from embryonic chick brain. Neuron 2:21-29.

Reinhold DS, Neet KE (1989) The lack of a role for protein kinase C in neurite extension and in the induction of ornithine decarboxylase by nerve growth factor in PC12 cells. J Biol Chem 264:3538-3544.

Richter-Landsberg C, Jastorff B (1986) The role of cAMP in nerve growth factor-promoted neurite outgrowth in PC12 cells. J Cell Biol 102:821-829.

Rydel RE, Greene LA (1988) cAMP analogs promote survival and neurite outgrowth in cultures of rat sympathetic and sensory neurons independently of nerve growth factor. Proc Natl Acad Sci USA 85: 1257-1261.

Tamaoki T, Nomoto H, Takahashi I, Yuzuru K, Morimoto M, Tomita F (1986) Staurosporine, a potent inhibitor of phospholipid/Ca ${ }^{++}$ dependent protein kinase. Biochem Biophys Res Commun 135:397402 .

Volk BW, Schneck L, eds (1975) Current trends in sphingolipidoses and allied disorders. New York: Plenum.

Winicov I, Gershengorn MC (1988) Sphingosine inhibits thyrotropinreleasing hormone binding to pituitary cells by a mechanism independent of protein kinase C. J Biol Chem 263:12179-12182.

Yankner BA, Shooter EM (1982) The biology and mechanism of action of nerve growth factor. Annu Rev Biochem 51:845-868. 